\title{
Vibrational properties of aluminum, nickel and copper surfaces
}

\author{
Peter D. Ditlevsen and Jens K. Norskov \\ Laboratory of Applied Physics, Technical University of Denmark, DK-2800 Lyngby, Denmark
}

Received 17 December 1990; accepted for publication 22 February 1991

\begin{abstract}
The vibrational properties of the (111) surface of aluminum and nickel and all the low-index surfaces of copper are examined. The surface phonon spectrum is calculated using the effective-medium theory to get the total energy and forces of the metallic system. A detailed discussion of the resulting dispersion relations and polarizations is presented together with a comparison to experimental data from electron energy loss and helium scattering experiments. Effective force constants at the surface are calculated and shown to differ from the bulk values in accordance with simple force constant model fits to experiment. We show analytically how these effective force constant changes are a consequence of the many-body character of the inter-atomic interactions in a metallic system.
\end{abstract}

\section{Introduction}

The inter-atomic interactions at the surface of a metal are in principle different from those in the bulk of the material. This is important for understanding reconstructions and relaxations of clean surfaces, but also for adsorbate-induced reconstructions, surface diffusion, roughening, and premelting. The simplest way of measuring these differences is by studying the surface phonons. This has been done quite extensively using inelastic helium scattering [1-3] and inelastic electron scattering $[4,5]$ techniques. The experimental data are usually interpreted using central-force models, and in general one finds that one has to change the inter-atomic force constants from the bulk values to get a reasonable fit. Such fitting schemes are never unique because two different fitting procedures with different range of the interactions, for instance, may give very different results for the force constants. The prime example in this context is the two different fits that have been proposed for the $\mathrm{Cu}(111)$ surface. One group reports that the coupling constant $k_{11}$ within the first layer should be decreased by $15 \%$ with respect to the bulk value [5]. Contrary to this it has been proposed that the coupling $k_{12}$ between the first and second layer is only $40 \%$ of the bulk value, and that interactions out to 6th-nearest neighbors are necessary for a proper description [6].

There is therefore a need for an analysis of the surface phonons using a treatment where the many-body nature of the interactions in a metal is properly included and where at the same time the effective force constants can be calculated. Nelson et al. [7] have applied the embedded-atom method to a study of the (100) and (111) surfaces of copper and the (111) surface of silver. They conclude that the method can give a good description of the measured relations and that the changes in effective force constants are modest. The noble metal (111) surfaces have also been studied by Jayanthi et al. [8] using the semi-empirical glue model.

In the present paper we apply another approximate total energy method, the effectivemedium theory, to the problem of calculating the surface phonons of metals. We study the (111) surfaces of aluminum and nickel and all the lowindex surfaces of copper. Dispersion relations as well as polarizations are calculated and compared to experiment. Very good agreement with experiment is found without fitting to any of the surface properties. The effective-medium theory is qualitatively similar to the embedded-atom method, and where a comparison is possible the present results 
agree with those of ref. [7]. In particular we also find modest changes in effective inter-atomic forces at the surface. The strength of the effective-medium theory in this context is that the terms entering the total energy expression have a clear physical interpretation, and we can therefore analytically derive and understand the physical origin of the force constant changes. Furthermore, the simplicity of the energy expression enables us to calculate the full dynamical matrix for the entire Brillouin zone, in contrast to the frozen phonon approach, taken by Ho and Bohnen [13], for calculation of the dispersion relations at the zone center and the zone boundary of aluminum from $a b$ initio energy calculations.

The paper is organized as follows. First, in section 2, we give a summary of the effectivemedium theory and give the parameters used in the present calculation. In section 3 we discuss the calculation of the dynamical matrix and the resulting phonons, and compare the calculated spectra to experiment. Section 4 contains the discussion of the origin of the force constant changes at the surface. Finally, in section 5 , we give a brief summary of the obtained results.

\section{Effective-medium theory}

The vibrational properties of a solid is determined by the second and (to a lesser degree) higher derivatives of the energy with respect to the atomic positions. The total energy of such a system can in principle be calculated from first principles in the adiabatic approximation. Calculating the total energy of a low-symmetry system, like a surface, is, however, computationally an enormous task. When the system involves hundreds of atoms per unit cell it is necessary to invoke simplifications in the energy calculation. It is also often advantageous to simplify such calculations in order to gain insight into the physics of the problem. The effective-medium theory is an approximate scheme for calculating the total energy of a metallic system $[9,10]$. The philosophy of the effective- medium theory is to view the atom as embedded in the homogenous electron gas consisting of the electron density tails from the neighboring atoms. It is a reflection of the observation that the energy of an atom in a metal depends primarily on the local environment set up by the surrounding atoms. The electrons screen very efficiently the long range interactions. The conjecture is then that the cohesive energy of a metallic system is to a first approximation, given by the sum

$E_{\text {cohesive }}=\sum_{i} E_{\mathrm{c}, i}\left(\bar{n}_{i}\right)$,

where $E_{\mathrm{c}, j}\left(\bar{n}_{i}\right)$ is the energy contribution from embedding the $i$ th atom in a homogeneous electron gas of density $\bar{n}_{i}$. The embedding density is the sum of the average of the electronic density tails $\Delta n_{j}$ of the neighboring atoms,

$\bar{n}_{i}=\sum_{j \neq i}\left\langle\Delta n_{j}\right\rangle_{i}$

The average is taken over the region of space occupied by the atom $i$. This first approximation of the cohesive energy provides a very appealing picture of the metallic system. The cohesive energy $E_{\mathrm{c}, i}\left(n_{i}\right)$ of an atom $i$, is a universal function of the embedding density $n_{i}$ and the atomic number of the atom. The dependence on the local environment enters through the embedding density $n_{i}$.

The energy $E_{\mathrm{c}}(\bar{n})$ of an atom embedded in a homogenous electron gas (jellium) can be obtained in the Kohn-Sham scheme of density functional theory [11]. It is composed by several parts:

$E_{\mathrm{c}}=E_{\mathrm{kin}}+E_{\mathrm{e}-\mathrm{e}}+E_{\mathrm{x}-\mathrm{c}}+E_{\mathrm{e}-\mathrm{l}}-E_{\text {jellium }}-E_{\mathrm{atom}}$.

The first three terms are the kinetic, Hartree and exchange-correlation energies of the electrons. $E_{\mathrm{e}-1}$ is the energy of the electrons in the electrostatic potential $\phi$ of the atom. When calculating the energy $E_{\text {hom }}$ of the atom in the homogeneous electron gas there is an additional energy term. This is the electrostatic energy of the positive jellium background in the atomic potential $\phi$. This term is not present in the real metallic system. In order to obtain the binding energy of the atom in 
the real metal it must be subtracted from $E_{\text {hom }}$ and we get,

$$
E_{\mathrm{c}}(\bar{n})=E_{\mathrm{hom}}(\bar{n})-\int \mathrm{d} r \phi(r) \bar{n} \equiv E_{\mathrm{hom}}(\bar{n})-\alpha \bar{n} .
$$

Since $\bar{n}$ is built up by the electron density tails from the neighboring atoms, the last integral can equally well be viewed as the electrostatic energy contribution from the electron density tails, of the neighboring atoms interacting with the atomic potential $\phi$ of the atom in question. Fig. 1 shows $E_{\mathrm{c}}(\bar{n})$ calculated for aluminum, nickel and copper [9]. From this calculation a very simple picture of cohesion in metals emerge. Consider the embedding density $\bar{n}$ as some (decreasing) function of the inter-atomic distance. The minimum of the $E_{\mathrm{c}}(\bar{n})$ curve determines the ground state embedding density, $n_{0}$, and thus the lattice constant. The curvature at the minimum is closely related to the bulk modulus of the crystal and the depth of the minimum gives the cohesive energy.

\subsection{The cohesive energy}

The cohesive encrgy functions $E_{\mathrm{c}}(\bar{n})$ shown in fig. 1 are conveniently parameterized by a polynomial as function of the embedding density.

$$
E_{\mathrm{c}}(\bar{n})=E_{0}+E_{2}\left(\frac{\bar{n}}{n_{0}}-1\right)^{2}+E_{3}\left(\frac{\bar{n}}{n_{0}}-1\right)^{3} \text {. }
$$

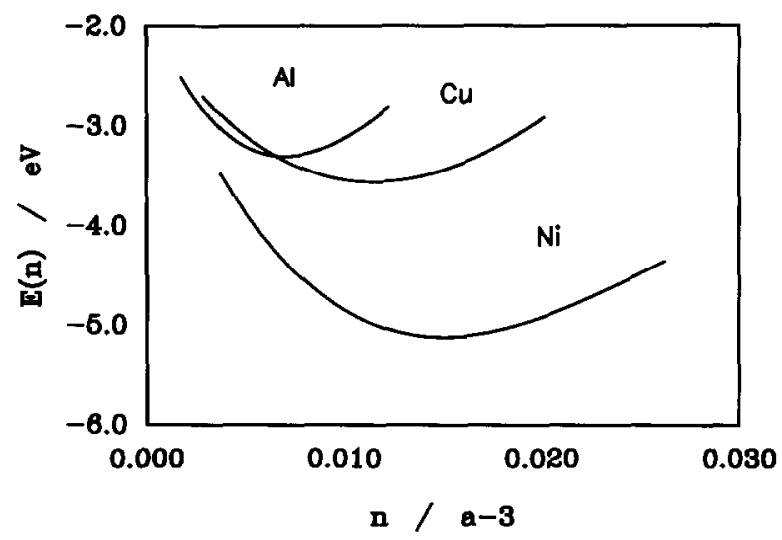

Fig. 1. The cohesive energy as a function of the embedding density for aluminum, nickel and copper.
The embedding density, $\bar{n}=\sum \Delta \bar{n}_{j}$, depends on the positions of the neighboring atoms. The form, eq. (1), of the cohesive energy show that manybody interactions play a crucial role in describing cohesion in a metal. Only if $E_{c}(\bar{n})$ had been a linear function would the density of eq. (2) give the cohesive energy as a sum of pair potentials.

\subsection{Correction terms}

The form, eq. (1), of the energy of a metallic system can be derived through well-defined approximations [9]. There will be correction terms to this expression,

$E=\sum_{i} E_{\mathrm{c}}\left(\bar{n}_{i}\right)+$ correction terms

The embedding density $\bar{n}$ is the sum of the average of the electron density tails of the neighboring atoms at the position of the atom (eq. (2)). The average is taken over a sphere centered at the nucleus of the atom with radius such that it contains $Z$ electrons, $Z$ being the nuclear charge of the atom. This is called the neutral sphere, and in a perfect fcc crystal this is just the Wigner-Seitz sphere. In the perfect fcc crystal the Wigner-Seitz cells are to a good approximation described as non-overlapping and space-filling spheres. This is the atomic-sphere approximation. In this approximation there will be no electrostatic interaction between the Wigner-Seitz spheres since they are neutral. When the metal is not in the perfect fcc structure the atomic-sphere approximation no longer holds. There will be an electrostatic correction term $E_{\mathrm{AS}}$, called the atomic-sphere correction. $E_{\mathrm{AS}}$ can be split up into contributions from each atom [9]:

$E_{\mathrm{AS}}=\sum_{i} E_{\mathrm{AS}}(i)$.

The term $E_{\mathrm{AS}}(i)$ can be written as the term $\alpha \bar{n}$, introduced in eq. (4), and a sum of pair-potentials:

$E_{\mathrm{AS}}(i)=\alpha \bar{n}_{i}-\sum_{j \neq i} V\left(r_{i j}\right)$.

In the fcc lattice this term should vanish, so the pair-potentials are simply parameterized by the 
embedding density that is obtained in the perfect fcc lattice,

$E_{\mathrm{AS}}(i)=\alpha\left(\bar{n}_{i}-\bar{n}_{i}^{\mathrm{fcc}}\right)$

The atomic-sphere correction can be viewed as the electrostatic energy from the repulsion of the overlapping regions of the neutral spheres, see fig. 2 .

In metals like the transition metals with a narrow d-band at the Fermi-level there is a one-electron correction term $E_{1 e}$. This contribution comes mainly from the d-d hybridization. The $E_{1 \mathrm{e}}$ term is usually small for aluminum, since there are no $\mathrm{d}$-bands, and for copper, since the d-bands are filled. For nickel this term gives a contribution due to the unfilled d-band [9]. The form of the one-electron term plays no significant role for the vibrational properties and it will be incorporated indirectly through the parameterization of the $E_{\mathrm{c}}$ function as discussed later in this paper. The total energy of the metal is then, simply,

$E=\sum_{i}\left[E_{\mathrm{c}}\left(\bar{n}_{i}\right)+E_{\mathrm{AS}}(i)\right]$,

where the sum is over the atoms of the system.

\subsection{The embedding density}

The way of choosing the embedding densities is outlined in the following. The embedding density $\bar{n}_{i}$ of an atom $i$ is the average over the neutral sphere $a_{i}$ of the density tails of the neighboring atoms. The electron density of an atom must be taken to be the density obtained from embedding the atom in the homogeneous electron gas. The induced charge density of an atom in the homogeneous electron gas of density $\bar{n}$ is, as described previously, calculated using the Kohn-Sham scheme of density functional theory. The relation

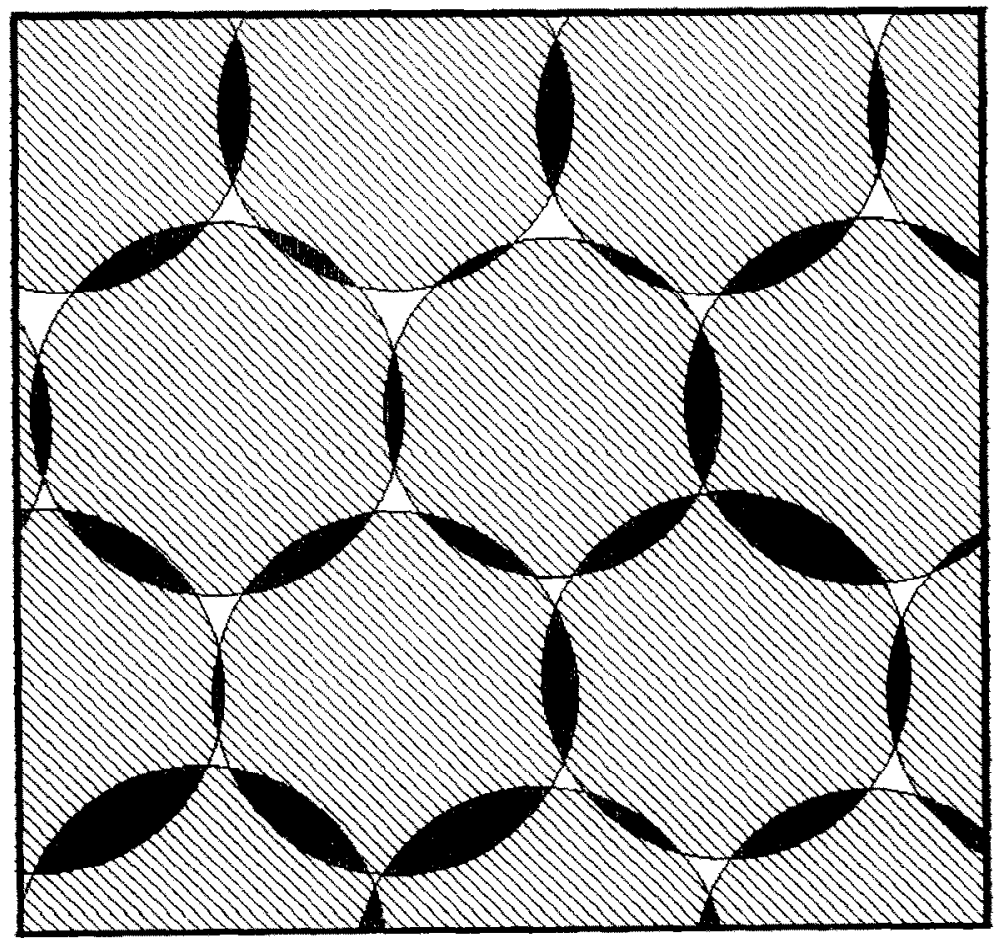

Fig. 2. The atomic-sphere correction to the energy comes from the electrostatic repulsion between the overlap regions of the neutral spheres. 
between the density $\bar{n}$ and the neutrality radius $s$ can to a good approximation be parameterized by an exponential,

$\bar{n}(s)=n_{0} \exp \left[-\eta\left(s-s_{0}\right)\right]$,

where the $n_{0}$ is the density at which the minimum value of the energy is obtained, (see fig. 1), $s_{0}$ is the corresponding Wigner-Seitz radius.

In general the average density $\Delta \bar{n}_{i j}$, of the electronic tail of atom $j$, averaged over the neutral sphere of radius $s_{i}$ around atom $i$ depends on the inter-atomic distance $r_{i j}$ and the radius $s_{i}$ of the sphere over which the average is taken. This dependence is also parameterized by an exponential,

$\Delta \bar{n}\left(r_{i j}, s_{i}\right)=\Delta \bar{n}_{0} \exp \left(-\eta_{2} r_{i j}+\eta_{1} s_{i}\right)$.

The sum of the average tail densities of the neighboring atoms inside the neutral sphere $a_{i}$ must be equal to the density corresponding to atom $i$ outside the neutral sphere. This self-consistency requirement on the density determines the radius of the ncutral sphere. For the perfect fec structure with Wigner-Seitz radius $s$ this implies

$\bar{n}(s)=\sum_{j} \Delta \bar{n}(r=\beta s, s)=12 \Delta \bar{n}(r=\beta s, s)$.

$\beta=(16 \pi / 3)^{1 / 3} / \sqrt{2}$ is the ratio of the nearestneighbor distance and the Wigner-Seitz radius in an fcc crystal. Then eqs. (11) and (12) imply the relation, $\eta=\beta \eta_{2}-\eta_{1}$. In the fcc lattice the summation over neighboring atoms is taken to be over the 12 nearest neighbors. Then eq. (12) can be rewritten,

$\Delta \bar{n}(r, s)=\frac{n_{0}}{12} \exp \left[-\eta_{2}\left(r-\beta s_{0}\right)+\eta_{1}\left(s-s_{0}\right)\right]$.

The embedding density $\bar{n}_{i}$ of atom $i$ can now be determined by solving the self-consistency equation (13) in general with respect to $s_{i}$. The result is:

$\bar{n}_{i}=n_{0}\left\{\frac{1}{12} \sum_{j} \exp \left[-\eta_{2}\left(r_{i j}-\beta s_{0}\right)\right]\right\}^{\eta /\left(\eta+\eta_{1}\right)}$.

The density $\bar{n}^{\mathrm{fcc}}$ entering eq. (9) is:

$\bar{n}_{i}^{\mathrm{fcc}}=\frac{n_{0}}{12} \sum_{j} \exp \left[-\eta\left(r_{i j} / \beta-s_{0}\right)\right]$.

\subsection{Elastic properties}

The elastic properties of a material is determined by the response to deformations away from the equilibrium configuration. The bulk modulus is given by

$B=V \frac{\partial^{2} E}{\partial V^{2}}=\frac{1}{12 \pi s_{0}} \frac{\partial^{2} E}{\partial s_{0}^{2}}$,

wherc $s_{0}$ is the Wigncr-Seitz radius. This corresponds to a uniform volume change of the fcc crystal. It will therefore only depend on the $E_{\mathrm{c}}$ part of the energy. The bulk modulus is thus determined by the curvature of the $E_{\mathrm{c}}(\bar{n})$ function in fig. 1. From eqs. (15) and (5) the result is,

$B=\frac{E_{2} \eta^{2}}{6 \pi s_{0}}$.

The other elastic constants are obtained in a similar manner. The $C_{44}$ is the shear modulus, it can be evaluated as the force constant of a volume conserving deformation, $\xi$, in the $\langle 100\rangle$ direction,

$$
\begin{aligned}
C_{44}= & \frac{\sqrt{2}}{a^{2}} \frac{\partial^{2} E}{\partial \xi^{2}} \\
\frac{\partial^{2} E}{\partial \xi^{2}}= & \frac{\mathrm{d}^{2} E}{\mathrm{~d} \bar{n}^{2}}\left[\frac{\mathrm{d} \bar{n}}{\mathrm{~d} \xi}\right]^{2}+\frac{\mathrm{d} E}{\mathrm{~d} \bar{n}} \frac{\mathrm{d} \bar{n}^{2}}{\mathrm{~d} \xi^{2}}+\frac{\mathrm{d}^{2} E}{\mathrm{~d} \bar{n}_{\mathrm{fcc}}^{2}}\left[\frac{\mathrm{d} \bar{n}_{\mathrm{fcc}}}{\mathrm{d} \xi}\right]^{2} \\
& +\frac{\mathrm{d} E}{\mathrm{~d} \bar{n}_{\mathrm{fcc}}} \frac{\mathrm{d} \bar{n}_{\mathrm{fcc}}^{2}}{\mathrm{~d} \xi^{2}} \\
= & \frac{\mathrm{d} E_{\mathrm{AS}}}{\mathrm{d} n} \frac{\mathrm{d} \bar{n}^{2}}{\mathrm{~d} \xi^{2}}+\frac{\mathrm{d} E_{\mathrm{AS}}}{\mathrm{d} n_{\mathrm{fcc}}} \frac{\mathrm{d} \bar{n}_{\mathrm{fcc}}^{2}}{\mathrm{~d} \xi^{2}}
\end{aligned}
$$

The last equality follows since $\mathrm{d} \bar{n} / \mathrm{d} \xi$ and $\mathrm{d} \bar{n}_{\mathrm{fcc}} / \mathrm{d} \xi$ vanishes for a volume conserving distortion and $\mathrm{d} E_{\mathrm{c}} / \mathrm{d} \bar{n}=0$ in the ground state. Thus the cohesive part of the energy is independent of such a deformation and $C_{44}$ depend solely on the atomic-sphere part of the energy, $E_{\mathrm{AS}}$. From eq. (19) we get,

$C_{44}=\frac{\alpha n_{0} \eta \eta_{1}}{16 \pi s_{0}}$.

\subsection{Effective-medium theory parameters}

The parameters of the theory can be calculated in the density functional formalism as outlined in 
Table 1

Comparison of the calculated an experimental values of lattice constants, bulk moduli, cohesive energies [12] and maximum bulk phonon frequencies $[9,13,3,4]$ (the numbers in the brackets are the frequency of the transversal phonon in the $M$ point for aluminum)

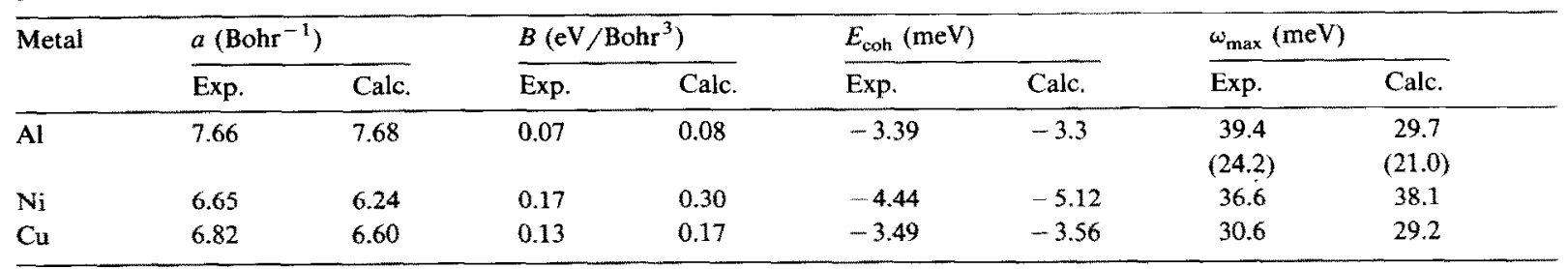

the previous sections. They only depend on the atomic number. The only input parameter that can not be obtained reliably from the calculation in the homogeneous electron gas is $\eta_{1}$. This is usually determined from the experimental value of $C_{44}$ through eq. (20). Using $\eta_{1}$ determined in this way the lattice constants, bulk moduli and cohesive energies can be calculated in the effectivemedium theory scheme. The agreement with experimental values are within typically $5-10 \%$, as is seen in table 1.

The good agreement between the theory and experimental values of the material parameters is a confirmation of the validity of the approximation scheme used in the effective-medium theory. The rather poor determination of the bulk modulus for nickel seen in table 1 is mainly a consequence of the omission of contribution to the total energy from the one-electron correction term.

The experimental bulk phonon dispersions for aluminium show a significant deviation from what would be expected from a simple nearest-neighbor force constant model. In such a model there is a simple $\sqrt{2}$ relation between the longitudinal and the transversal zone-boundary phonon frequency in the $M$ point. The numbers in bracket in table 1 are the experimental and calculated frequencies of the transversal zone-boundary phonon for aluminum. This phonon is more closely related to the $C_{44}$ than the longitudinal zone-boundary phonon and closer agreement between theory and experiment is observed.

The surface vibrational properties depend upon changes in the force-fields of 5-10\% at the surface compared to the bulk. This is the same order of magnitude as the approximations implied by the effective-medium theory. When studying these effects quantitatively it is preferable to fit the parameters so that the bulk is precisely described. The fitting procedure is ambiguous since only the products $E_{2} \eta^{2}$ and $\alpha \eta \eta_{1}$ occur in $B$ and $C_{44}$, respectively. The fitting is chosen to be the following. The electronic density $\bar{n}(s)$ is obtained from the calculation through eq. (11). This determines the parameter $\% . s_{0}$ is fitted by the experimental value of the lattice constant and $E_{2}$ is fitted to

Table 2

The effective-medium theory parameters for aluminum, nickel and copper. In the first columns ("Calc.") only $\eta_{1}$ is fitted (to $C_{44}$ ), in the second columns ("Fitted") $s_{0}$ is fitted to the lattice constant, $E_{2}$ to the bulk modulus, and $\eta_{1}$ to the bulk phonon frequency

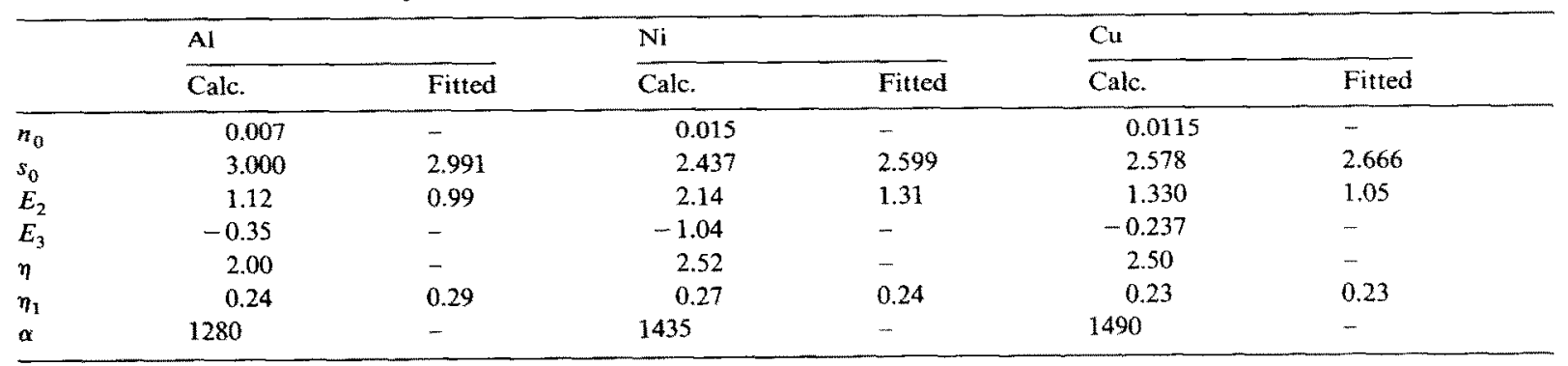


give the experimental value of the bulk modulus by the use of eq. (18). $\eta_{1}$ is then fitted to give the experimental maximum bulk phonon frequency in a harmonic calculation. For aluminum the transversal phonon frequency in the $M$ point is used for fitting. How to determine the frequencies from the energy expression is the topic of the following section. The fitted parameters are listed in table 2.

The relatively big changes in the parameter $E_{2}$ for nickel, due to the fitting, is mainly a consequence of the omission of the $E_{1 \mathrm{e}}$ term in the energy expression. The fitting procedure is another indirect way of incorporating the effect of d-band hybridization. The qualitative aspects of the vibrational physics is described equally well both in bulk and at the surface by the two parameter sets. But in order to compare the calculations with experiments the fitted parameter set will be used in the rest of this work.

\section{Harmonic theory}

The vibrational eigenstates of surfaces have been calculated in the harmonic approximation. The components of the dynamical matrix are given by the second derivative of the total energy of the system with respect to the atomic coordinates. At a surface the translational symmetry of the lattice is absent in the direction perpendicular to the surface. The surface phonon modes are therefore generalized plane waves parallel to the surface with a two-dimensional parallel crystal momentum. The dynamical problem is solved for a slab, so thick that the two surfaces do not interact. This reduces the dynamical problem to a finite matrix problem. Only near the $\bar{\Gamma}$-point, where the wavelength of the phonon is comparable to the thick- ness of the slab is hybridization between the surface states on the two surfaces observed. A 30-layer slab turns out to be sufficient for convergence in most of the Brillouin-zone. The eigenstates $\epsilon_{\sigma}^{\mathrm{a}}$ of the dynamical equation are $\left(3 \times N_{z}\right)$ dimensional polarization vectors, $N_{z}$ is the number of layers in the slab, and $\sigma$ is the polarization index. The dynamical matrix is symmetrical so that the eigenvalues are real, but the eigenvectors are in general complex corresponding to elliptic motion of the atoms in the surface. When calculating the dynamical matrices the surface layers are relaxed to the equilibrium positions. The surface relaxations are shown in table 3 .

\subsection{The copper (100) and (110) surfaces}

Careful experimental measurements of the surface phonons of $\mathrm{Cu}(100)$ [1] and $\mathrm{Cu}(110)$ [2] has been performed. Calculated and experimental values of surface phonon frequencies at the zone boundaries are summarized in table 4 , and the full dispersion relations along high symmetry directions are shown in fig. 3 . On the (100) surface there is only one surface phonon outside the bulk and in the $\bar{\Gamma}-\bar{M}$ direction. It is the Rayleigh mode, for which the calculation is in good agreement with the experimental data. In the $\bar{M}$ point the polarization of the Rayleigh mode is particularly simple. In a nearest-neighbor central-force model it is a vibration strictly in the first layer and the ratio of the frequency of the Rayleigh mode to the frequency at the bottom of the bulk band is $1 / \sqrt{2}$. The Rayleigh mode calculated from the simple nearest-neighbor central-force model is indicated by the dashed curve. The deviation of the

Table 3

The first- and second-layer surface relaxation of the low-index surfaces, comparison between calculation and L.FED experiments for aluminum [14], nickel [15] and copper [16] (the relaxations are in percent of the inter-atomic distance)

\begin{tabular}{|c|c|c|c|c|c|c|c|}
\hline & & $\mathrm{Al}$ & & $\mathrm{Ni}$ & & $\mathrm{Cu}$ & \\
\hline & & Calc. & Exp. & Calc. & Exp. & Calc. & Exp. \\
\hline (111) & $\Delta d_{12}$ & -1 & 0.9 & -1 & $-1.2 \pm 1.2$ & -1 & - \\
\hline$(100)$ & $\Delta d_{12}$ & -3 & 0.0 & -2 & $-1.1 \pm 1.1$ & -1 & $-1.1 \pm 0.4$ \\
\hline (110) & $\Delta d_{12}$ & -6 & $-8.6 \pm 0.8$ & -5 & $-4.8 \pm 1.6$ & -3 & $-8.5(-5.3)^{a)}$ \\
\hline & $\Delta d_{23}$ & 1 & $5.0 \pm 1.1$ & 1 & - & 0 & $2.3(3.3)^{a)}$ \\
\hline
\end{tabular}

\footnotetext{
a) HEIS measurements [16].
} 
Table 4

Calculated and experimental values of the frequencies of the surface phonons at the zone boundaries of $\mathrm{Cu}(110)$ and $\mathrm{Cu}(100)$ (the energies are in $\mathrm{meV}$ )

\begin{tabular}{|c|c|c|c|c|c|}
\hline \multirow[t]{2}{*}{$(110)$} & \multicolumn{2}{|l|}{$\overline{\bar{X}}$} & \multicolumn{3}{|l|}{$\overline{\mathrm{Y}}$} \\
\hline & $\overline{S_{1}}$ & $\overline{S_{7}}$ & $\overline{S_{1}}$ & $\mathrm{~S}_{3}$ & $\mathrm{~S}_{5}$ \\
\hline \multirow{4}{*}{$\begin{array}{l}\text { Calc. } \\
\text { Exp. }{ }^{\text {a) }} \\
(100)\end{array}$} & 13.5 & 24.2 & 9.7 & 12.4 & 19.7 \\
\hline & & 24.0 & & & \\
\hline & $\overline{\mathbf{M}}$ & & $\overline{\mathrm{X}}$ & & \\
\hline & $S_{1}$ & & $\mathbf{S}_{1}$ & $S_{4}$ & $\mathrm{~S}_{7}$ \\
\hline \multirow{2}{*}{$\begin{array}{l}\text { Calc. } \\
\left.\text { Exp. }^{b}\right)\end{array}$} & 16.3 & & 11.4 & 13.4 & 25.4 \\
\hline & 16.3 & & & 12.9 & \\
\hline
\end{tabular}

a) Taken from ref. [2].

b) Taken from ref. [1].

full calculation from the $1 / \sqrt{2}$ relation is a consequence of the change of the inter-atomic forces in the surface.

On the $\mathrm{Cu}(110)$ surface the dispersions along the direction perpendicular to the close-packed rows are shown. A very detailed discussion of the $\mathrm{Cu}(110)$ surface phonon spectrum has been presented by Zeppenfeld et al. [2]. Near the $\bar{Y}$ point there are three bands below the bulk projected band. Near the $\bar{\Gamma}$ point the two become surface resonances. At the $\bar{Y}$ point the lowest lying surface phonon is longitudinal in the first surface layer

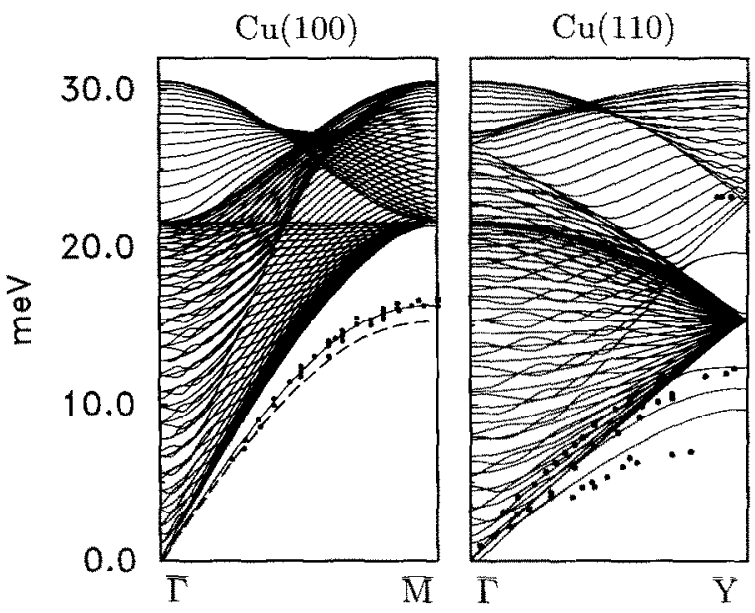

Fig. 3. The dispersion curves for $\mathrm{Cu}(100)$ [1] and $\mathrm{Cu}(110)$ [2]. Experiments are helium scattering. The dashed curve on the $\mathrm{Cu}(100)$ indicates the dispersion curve expected from a nearest-neighbor central-force model.

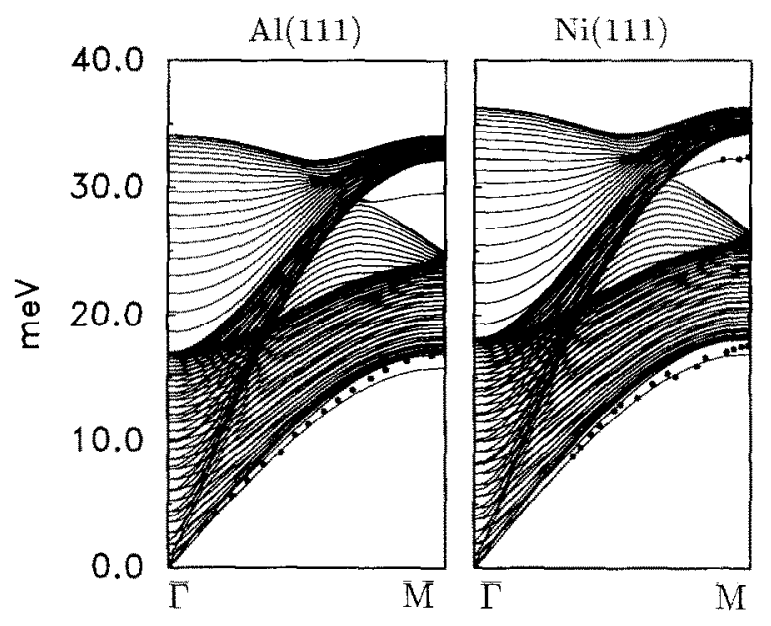

Fig. 4. Calculated and experimental dispersion curves for $\mathrm{Al}(111)$ [17] and $\mathrm{Ni}(111)$ [4]. The aluminum surface is examined by helium scattering, the nickel surface is examined by high-resolution EELS.

and polarized perpendicular to the surface in the second layer. This means that the close-packed rows move collectively forth and back while the second-layer atoms between the rows are pushed up and down. The discrepancy between the calculation and the experiment for this mode seen in fig. 3 originates from the approximation that only nearest neighbors contribute to the density sum in

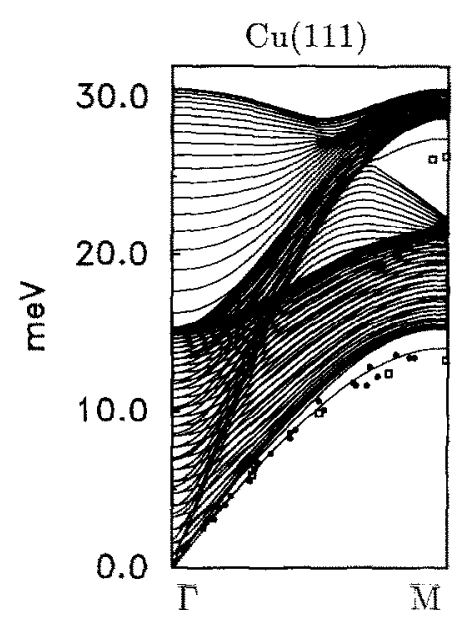

Fig. 5. A comparison of the calculated and experimental dispersion relations for the $\mathrm{Cu}(111)$ surface. The dots are deduced from helium scattering experiments [3] and the squares from EELS experiments [5]. 
Table 5

Comparison of the calculated and experimental values of the frequencies of the surface phonons at the zone boundaries of the (111) surfaces of aluminum, nickel and copper (the energies are in $\mathrm{meV}$ )

\begin{tabular}{|c|c|c|c|c|c|c|c|c|}
\hline & \multicolumn{4}{|l|}{$\overline{\mathbf{M}}$} & \multicolumn{4}{|l|}{$\overline{\mathbf{K}}$} \\
\hline & \multicolumn{2}{|l|}{$S_{1}$} & \multicolumn{2}{|l|}{$\mathrm{S}_{2}$} & \multicolumn{2}{|l|}{$S_{1}$} & \multicolumn{2}{|l|}{$\mathrm{S}_{3}$} \\
\hline & Calc. & Exp. & Calc. & Exp. & Calc. & Exp. & Calc. & Exp. \\
\hline $\mathrm{Al}^{\mathrm{a})}$ & 15.8 & 16.8 & 29.6 & - & 17.0 & 18.2 & 24.8 & - \\
\hline $\mathrm{Ni}^{\mathrm{b})}$ & 17.1 & 17.2 & 32.3 & 32.3 & 18.5 & - & 26.9 & - \\
\hline $\mathrm{Cu}$ & 14.0 & 13.4 & 27.4 & 26.3 & 14.9 & 14.4 & 22.8 & - \\
\hline
\end{tabular}

a) Experimental values taken from ref. [17]

b) Experimental values taken from ref. [4].

c) Experimental values taken from refs. [3,5].
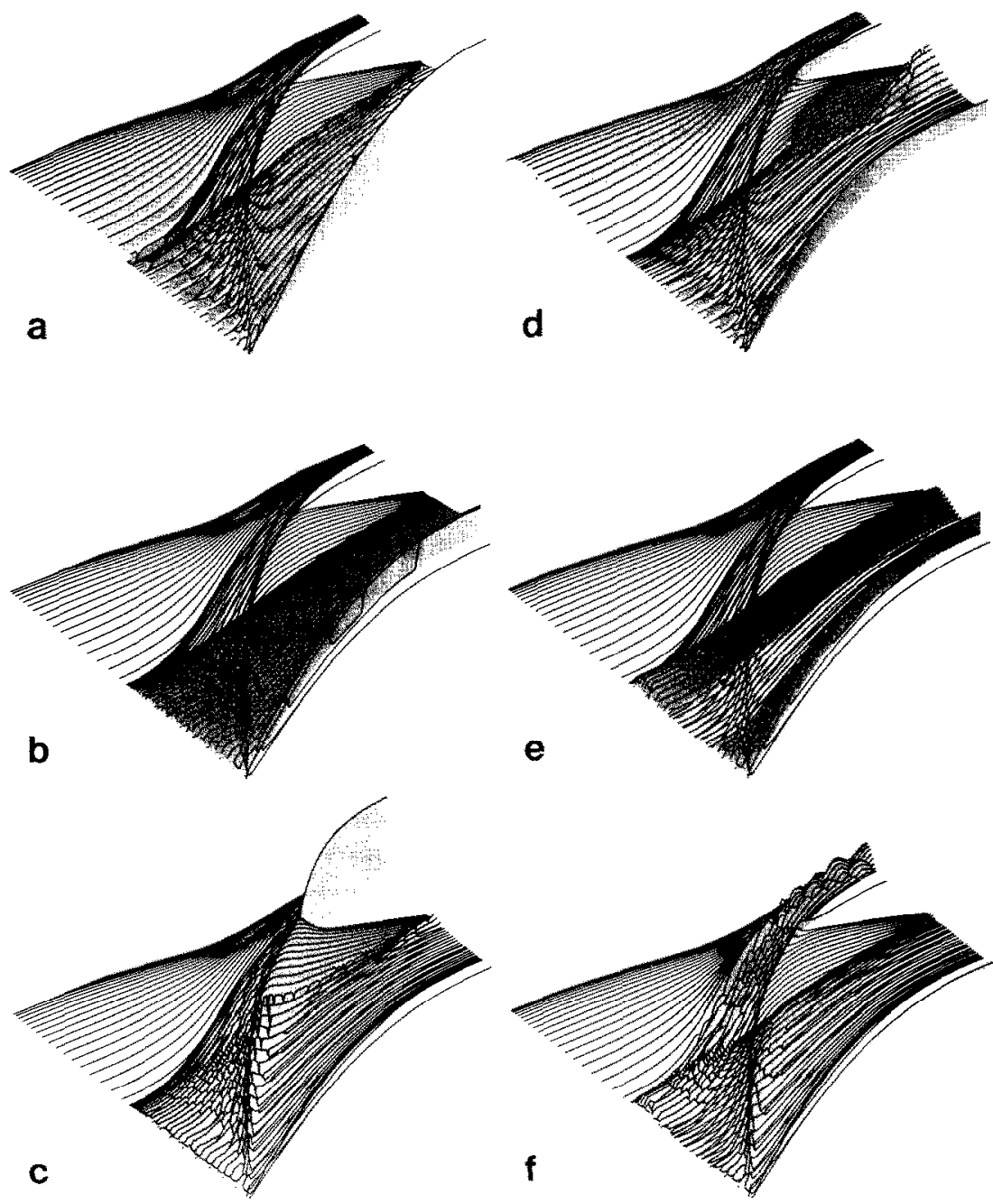

Fig. 6. The projected DOS in the first (left) and second (right) layer as a function of surface momentum and frequency in the $\bar{\Gamma}-\bar{M}$ direction. The top panels, (a) and (d), shows the transversal polarization perpendicular to the surface, the middle panels, (b) and (e), shows the shear polarization and the bottom panels, (c) and (f), shows the longitudinal polarization. 
eqs. (15) and (16). The first layer atoms are moving directly toward the second nearest neighbor atoms in the surface, therefore one expects the second nearest neighbor effects to be important in the determination of the frequency of that mode. The second phonon is concentrated in the first layer with in-plane transverse polarization. This means that the close-packed rows have a shear motion. The third phonon is the Rayleigh phonon, where the first layer moves perpendicular to the surface. The second layer has, as a consequence, in-plane longitudinal motion. Besides the three low-lying surface there is a higher-lying gap mode. This is mostly longitudinal.

\subsection{The (111) surfaces of aluminum, nickel and copper}

Calculated and experimental values of surface phonon frequencies at the zone boundaries are summarized in table 5 , and the full dispersion relations along the $\langle 211\rangle$ direction for $\mathrm{Al}(111)$ are shown in fig. 4. The dispersion relations of the Cu(111) surface are shown in fig. 5. The calculated dispersions are seen to be in good agreement with He-scattering and EELS experiments for all three metals. The deviations between the experiments and the calculation for the $\mathrm{Al}(111)$ surface is a reminiscence of the fact that the approximate method can not account for the anomalous rela- tion between the frequencies of the bulk longitudinal and transversal modes of aluminum as mentioned earlier. The effect of very-long-range interactions is not taken into account in this approximation where only the electronic density of the nearest neighbors is included in the energy and force calculation. More accurate calculations has been performed for the Al(110) surface by Eguiluz et al. [18] and it has been demonstrated that very-long-range interactions are important in the case of aluminum. The surface phonons of the $\mathrm{Cu}(111)$ surface have previously been calculated using the embedded-atom method [7]. This calculation is in good agreement with the results obtained from the effective-medium theory.

The projected density of states, for the whole $\bar{\Gamma} \cdot \bar{M}$ direction, is shown in fig. 6 . The projected DOS in layer $i$ and direction $\alpha$ is defined as $g_{i \alpha}(k, \omega)=\left|\epsilon^{i \alpha}(k)\right|^{2} \delta\left(\omega-\omega_{k}\right)$, where $\epsilon^{i \alpha}(k)$ is the eigenvector corresponding to the eigenfrequency $\omega_{k}$. The Rayleigh mode $\left(S_{1}\right)$ is a surface mode polarized perpendicular to the surface below the bulk band, it is mostly concentrated in the first few surface layers, see figs. $6 a$ and $6 \mathrm{~d}$. The lowering of the frequency of the Rayleigh mode, compared to the bulk transversal mode, is a consequence of the smaller coordination number of the surface atoms. The frequency of the Rayleigh mode is shifted up compared to what would be expected from a simple nearest-neighbor central-force model
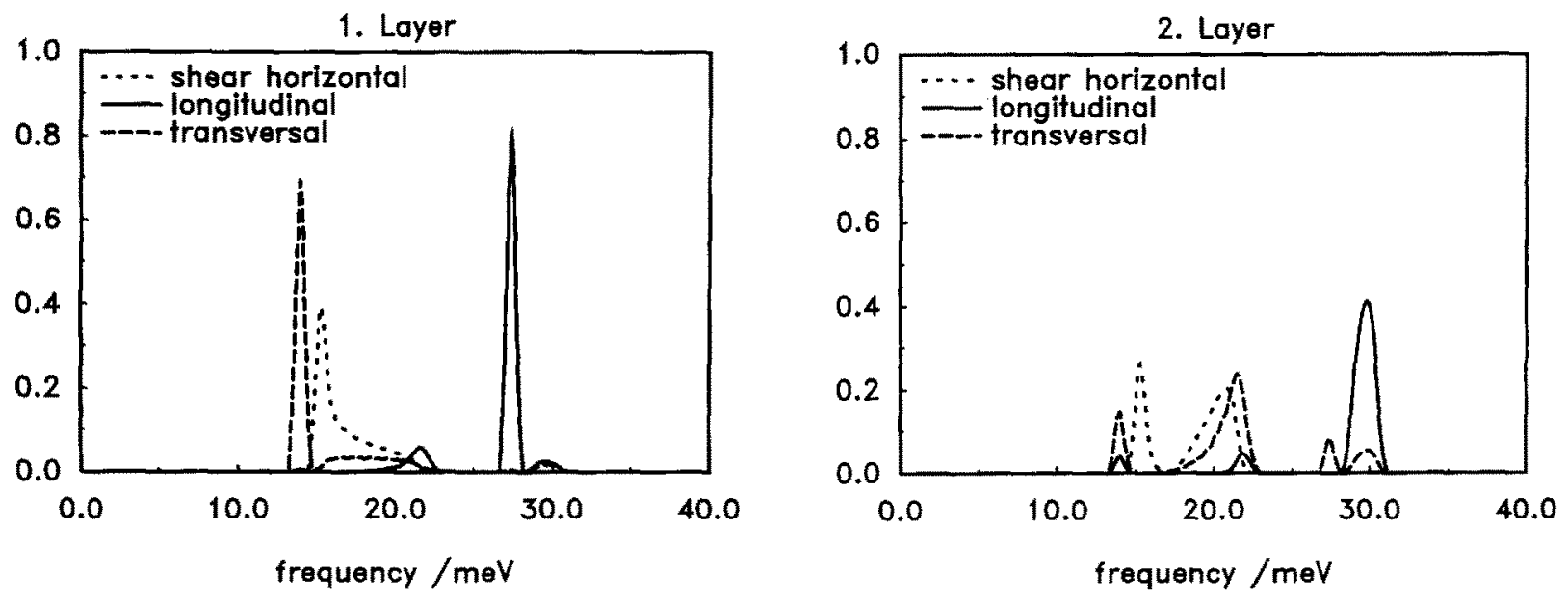

Fig. 7. The projected DOS in the first and second layer for $\mathrm{Cu}(111)$ at the $\bar{M}$ point. The lines has been broadened 0.5 meV by a Lorentzian as an aid for the eye. 
with only one force constant, $k_{i j}=k_{\text {bulk }}$. The shift of this mode is mainly governed by the forces between first and second layer atoms. The experimental value, $13 \mathrm{meV}$ at the $\overline{\mathrm{M}}$ point, can be fitted well by an increase in inter-layer force constant between first and second layer, $k_{12}$, of approximately $10 \%$ [5].

At the bottom of the projected bulk band there is a shear horizontal resonance with polarization in the surface plane, see fig. $6 \mathrm{c}$. From $\bar{\Gamma}$ to approximately $\frac{2}{3}$ of the way from $\bar{\Gamma}$ to $\bar{M}$, where a gap in the projected bulk band opens, there is a broad resonance. This resonance is in-plane polarized and mostly longitudinal, see fig. $6 \mathrm{~b}$. It has substantial weight in the first surface layer. In the gap this mode narrows into a longitudinal surface mode $\left(\mathrm{S}_{2}\right)$. The gap mode has most of its weight in the first surface layer. Below the gap there is an in-plane polarized resonance $\left(S_{6}\right)$ at approximately $21 \mathrm{meV}$ at the $\overline{\mathrm{M}}$ point, this resonance has very little weight on the first surface layer, and is mostly concentrated in the second and subsequent layers, see fig. $6 \mathrm{f}$. The projected density of state at the $\bar{M}$ point for the first and second atomic layer is shown in fig. 7 , it is seen that $S_{6}$ is a mixed resonance with second layer polarized both parallel and perpendicular to the surface at the $\overline{\mathbf{M}}$ point. The surface phonons and resonances are shown schematically in fig. 8 .

The atomic motion corresponding to the polarizations can be messy due to the rather complicated symmetry of the (111) face. The motion will in general be elliptical and the polarization vectors complex valued. An example is sketched in fig. 9.

The good agreement between the calculated

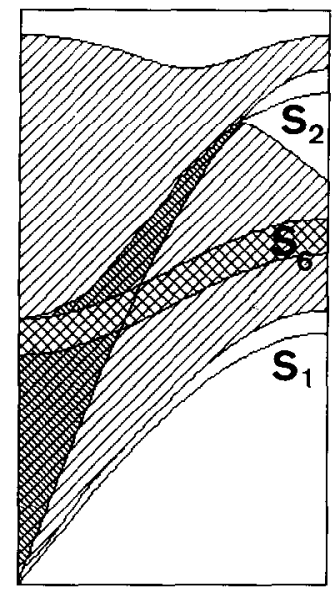

Fig. 8. The $\bar{\Gamma}-\bar{M}$ direction on the (111) surface. The hatched area in the bulk band indicates the surface resonances.

and measured dispersion relations are summarized in fig. 10. We have not made detailed calculations of the EELS intensities, but the changes in effective force constants we find are of the same order of magnitude as those used in a simple centralforce model calculation [5] and consequently the polarizations must be rather similar. The good detailed agreement between calculated and measured electron energy loss intensities found in ref. [5] can therefore be assumed also to hold for the present calculation of the phonons. Such calculations will, in the case of $\mathrm{Ni}(111)$, be presented elsewhere [19].

Regarding the helium scattering measurements, we show in fig. 11 a comparison of a distortedwave Born-approximation (DWBA) calculation of the time of flight spectrum with the measured one [20]. The DWBA calculation has been performed
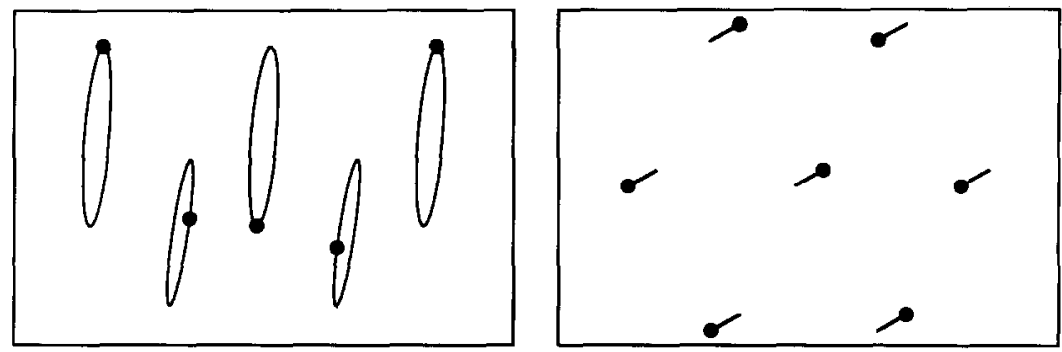

Fig. 9. Atomic motion (exaggerated) of the Rayleigh phonon at the $\bar{M}$ point of the $\mathrm{Cu}(111)$ surface. The left panel shows the motion of the first and second layer atoms in the $x z$-plane and the right panel shows the motion in the surface plane. 


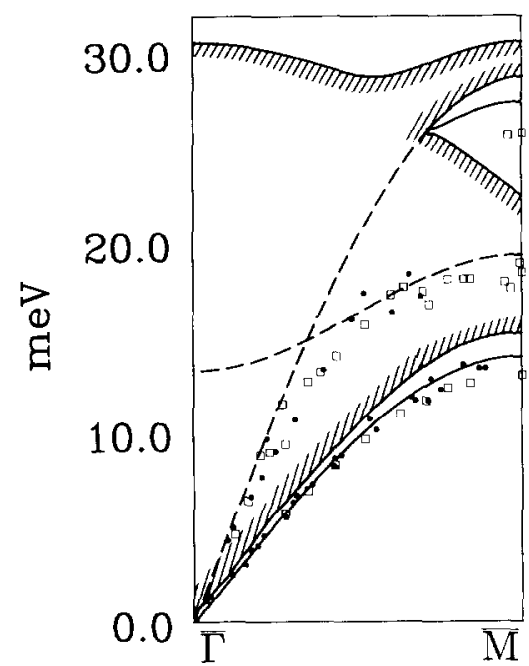

Fig. 10. Helium scattering [3] (dots) and EELS [5] (squares) measurements of the $\mathrm{Cu}(111)$ surface phonons and resonances.

The curves are the calculated dispersions.

according to the theory of Bortolani et al. [6]. It is clear that while the low-energy (Rayleigh mode) peaks are well described, the intensities of higherenergy peaks in the experiment are not reproduced by the theory. This may be due to an inadequate description of the phonons, or to a too simple description of the helium scattering process. We will discuss this further in a forthcoming paper [21].

\section{Inter-atomic forces at surfaces}

The interesting aspect of the the study of the surface phonons is the changes of the effective inter-atomic force constants due to the presence of the surface. This is what determines the shifts of the surface-induced modes in the phonon spectra, but also, of cause, the relaxations and in some cases reconstructions that occur at metal surfaces. We will therefore use this section to further understand the reason why the effective interactions changes from the bulk.

Given the total energy, $E(\{i\})$ of the metal as a function of atomic positions $\{i\}$ the effective force constants are defined in the following way. If the energy is a sum of harmonic pair-potentials, $\boldsymbol{\Phi}_{i j}=k_{i j}\left[\hat{\boldsymbol{R}}_{i j} \cdot\left(\boldsymbol{u}_{i}-\boldsymbol{u}_{j}\right)\right]^{2} / 2$, then the dynamical

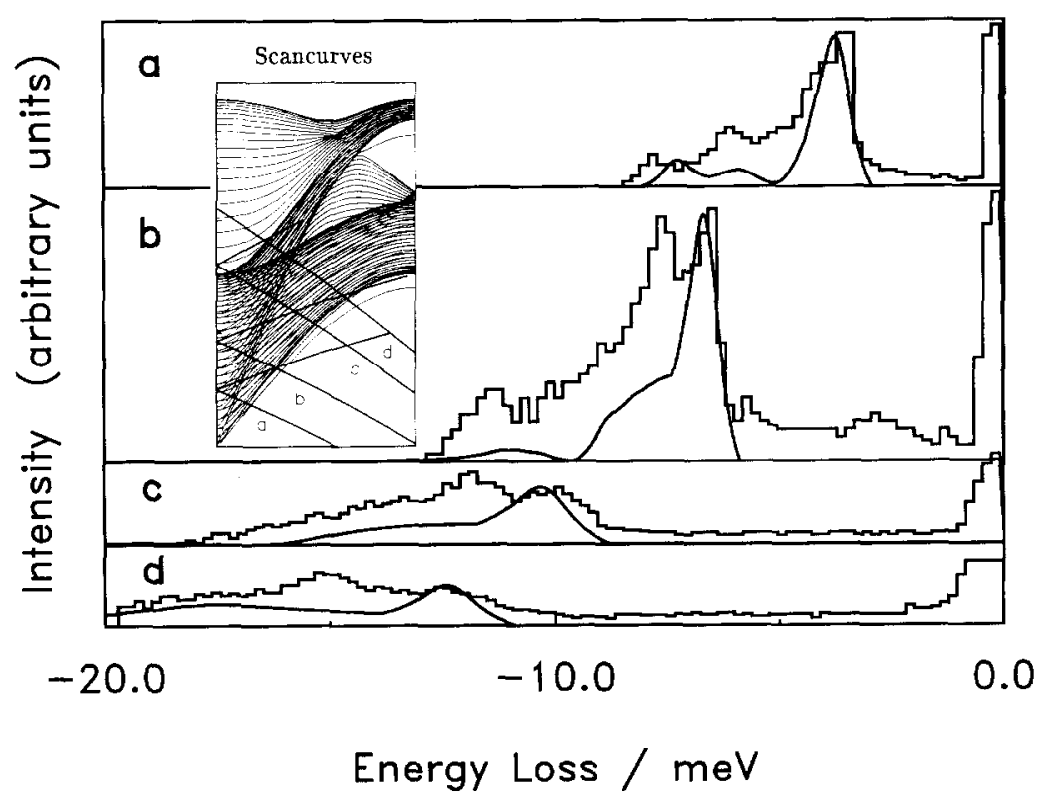

Fig. 11. A comparison of the experimental He TOF spectra (histogram) [3] with those calculated from the one-phonon distorted-wave Born-approximation (full curve). The curves are normalized to the experimental intensity of the Rayleigh mode, and the instrumental broadening is the one reported in ref. [3]. 
matrix is particularly simple, $D_{i j}^{\alpha \beta}=k_{i j} \hat{R}_{i j}^{\alpha} \hat{R}_{i j}^{\beta}$, and the inter-atomic force constant between atom $i$ and atom $j$ is,

$k_{i j}=D_{i j}^{x x}+D_{i j}^{y y}+D_{i j}^{z z}$.

This is the definition of the effective force constant where $D_{i j}^{\alpha \beta}=\partial^{2} E / \partial u_{i}^{\alpha} \partial u_{j}^{\beta}$ and $E$ is the effective-medium theory energy. The most important forces determining the surface phonon spectrum is the nearest-neighbor forces in the first layer, $k_{11}$, and the nearest-neighbor forces between first- and second-layer atoms, $k_{12}$, they are only moderately changed compared to the bulk value, $k_{\text {bulk }}$, at the (111) surfaces of aluminum, nickel and copper as shown in table 6 . This is very reasonable since these surface are close packed, and they do not reconstruct.

The change in the force constants at the surface of metals is a consequence of the many-body interactions in the metal. It can be rather simply understood in the effective-medium theory. The forces given by eq. (21) are proportional to the second derivative of the energy with respect to a particular deformation of the crystal. Consider a distortion $\xi$ corresponding to a zone boundary phonon, then the second derivative of the cohesive part of the energy, eq. (10), is given by,

$$
\frac{\mathrm{d}^{2} E_{\mathrm{c}}(n(\boldsymbol{r}))}{\mathrm{d} \xi^{2}}=\frac{\mathrm{d}^{2} E_{\mathrm{c}}}{\mathrm{d} n^{2}}\left[\frac{\mathrm{d} n}{\mathrm{~d} \xi}\right]^{2}+\frac{\mathrm{d} E_{\mathrm{c}}}{\mathrm{d} n} \frac{\mathrm{d}^{2} n}{\mathrm{~d} \xi^{2}} \text {. }
$$

In the bulk, at equilibrium, the cohesive energy, $E_{\mathrm{c}}(n)$, takes its minimum value and $\mathrm{d} E / \mathrm{d} n=0$. For a zone boundary phonon $\mathrm{d} n / \mathrm{d} \xi=0$ by symmetry, making both terms zero. $E_{\mathrm{AS}}$ thus determines the zone boundary phonons as described earlier. This will not be true at the surface. The $E_{\mathrm{AS}}$ term will still determine a large fraction of the effective force constants, but the contribution from

Table 6

Effective force constants in the surface layer and between firstand second-layer atoms on the (111) lace (the force constants are normalized with respect to the bulk force constant)

\begin{tabular}{lll}
\hline Metal & $k_{11}$ & $k_{12}$ \\
\hline $\mathrm{Al}$ & 0.89 & 1.04 \\
$\mathrm{Ni}$ & 0.90 & 1.10 \\
$\mathrm{Cu}$ & 0.95 & 1.09 \\
\hline
\end{tabular}

eq. (22) will no longer vanish. For a distortion parallel to the surface the first term in eq. (22) will still be zero due to symmetry, but for the atoms at the surface $\mathrm{d} E_{\mathrm{c}} / \mathrm{d} n$ is non-zero because the surface atoms have less nearest neighbors than the bulk atoms. The electron density that these atoms are embedded in is smaller than the optimum value $n_{0}$. From fig. 1 it follows that $\mathrm{d} E_{\mathrm{c}} / \mathrm{d} n$ is negative for the surface atoms. The force constant $k_{11}$ between atoms in the first layer is therefore smaller than in the bulk $k_{\text {bulk. }}$. For a distortion perpendicular to the surface this is still true, but here $\mathrm{d} n / \mathrm{d} \xi$ is no longer zero and the first term in eq. (22) will give a large and positive contribution to the force constant. Therefore the force constant between first- and second-layer atoms, $k_{12}$ is larger than $k_{\text {bulk }}$. This effect is not due to the inward relaxation of the surface but enhanced by it. It is the many-body properties of the $E_{\mathrm{c}}$ function that give this effect. A pair-potential description of a metal corresponds in the effective-medium theory to a linear $E_{c}(n)$ function. Since $n$ is a sum of contributions from the neighbors $E_{\mathrm{c}}(n)$ will only be a pair sum if it is linear in $n$.

\section{Summary}

We have applied the effective-medium theory to calculate the vibrational properties of the (111) surface of aluminum and nickel and all the low-index surfaces of copper. The calculated frequencies of the Rayleigh and gap modes are in good agreement with the values determined by helium and electron scattering for all surfaces. By calculating the local density of states of the polarization amplitudes in the surface layers, we have a clear picture of the surface phonon and resonance spectrum of the $\mathrm{Cu}(111)$ surface. The calculated polarizations of the $\mathrm{Cu}(111)$ surface accounts for the measured EELS spectra. The surface phonon spectrum is determined by the effective interatomic force constant at the surface. We have shown that the effective-medium theory provides a simple picture of the mechanisms governing the changes in the inter-atomic forces at the surface. These effects are consequences of the many-body nature of the interactions in the metal. 


\section{Acknowledgements}

Many helpful discussions with N. Chetty, K.W. Jacobsen, O.H. Nielsen, and P. Stoltze are gratefully appreciated. The research has been supported financially by the Danish Research Councils through the Center for Surface Reactions.

\section{References}

[1] M. Wuttig, R. Franchy and H. Ibach, Z. Phys. B 65 (1986) 71.

[2] P. Zeppenfeld, K. Kern, R. David, K. Kuhnke and G. Comsa, Phys. Rev. B 38 (1988) 12329.

[3] U. Harten, J.P. Toennies and Ch. Wöll, Faraday Disc. Chem. Soc. 80 (1985) 137.

[4] W. Menezes, P. Knipp, G. Tisdale and S.J. Sibener, Phys. Rev. B 41 (1990) 5648.

[5] M.H. Mohamed, L.L. Kesmodel, B.M. Hall and D.L. Mills, Phys. Rev. B 38 (1988) 5856

[6] V. Bortolani, A. Franchini, F. Nizzoli and G. Santoro, Phys. Rev. Lett. 52 (1984) 429;

G. Santoro, A. Franchini and V. Bortolani, preprint.

[7] J.S. Nelson, M.S. Daw and E.C. Sowa, Phys. Rev. B 40 (1989) 1465.

[8] C.S. Jayanthi, H. Bilz, W. Kress and G. Benedek, Phys. Rev. Lett. 59 (1987) 795.
[9] K.W. Jacobsen, J.K. Nørskov and M.J. Puska, Phys. Rev. B 35 (1987) 7423 .

[10] K.W. Jacobsen, Comments Condensed Maters. Phys. 14 (1988) 129.

[11] W. Kohn and L. Sham, Phys. Rev. 140 (1965) A1133.

[12] C. Kittel, Introduction to Solid State Physics (Wiley, New York, 1976).

[13] K.M. Ho and K.P. Bohnen, Phys. Rev. Lett. 56 (1986) 934.

[14] J.R. Noonan and H.L. Davis, Phys. Rev. B 29 (1984) 4349.

[15] J.E. Demuth, P.M. Marcus and D.W. Jepsen, Phys. Rev. B 11 (1975) 1460.

[16] D.L. Adams, H.B. Nielsen, J.N. Andersen, I. Stensgaard, R. Feidenhans'l and J.E. Sørensen, Phys. Rev. Lett. 49 (1982) 669:

H.L. Davis and J.R. Noonan, Surf. Sci. 126 (1983) 245.

[17] A. Lock, J.P. Toennies, Ch. Wöll, V. Bortolani, A Franchini and G. Santoro, Phys. Rev. B 37 (1988) 7087

[18] A.G. Eguiluz, A.A. Maradudin and R.F. Wallis. Phys. Rev. Lett. 60 (1988) 309.

[19] W. Menezes, P. Knipp, G. Tisdale, S.J. Sibener and P.D. Ditlevsen, to be published.

[20] P.D. Ditlevsen and J.K. Nørskov, in: Proc. of Vibrations at Surfaces VI, J. Electron Spectrose. Relat. Phenom. special edition.

[21] P.D. Ditlevsen, N. Chetty and J.K. Nørskov, to be published. 